\title{
Articulation and Liars
}

\author{
Sergi Oms \\ University of Barcelona \\ Logos, Research Group in Analytic Philosophy \\ BIBLID [0873-626X (2017) 46; pp. 383-399] \\ DOI: $10.1515 /$ disp-2017-0011
}

\begin{abstract}
Jamie Tappenden was one of the first authors to entertain the possibility of a common treatment for the Liar and the Sorites paradoxes. In order to deal with these two paradoxes he proposed using the Strong Kleene semantic scheme. This strategy left unexplained our tendency to regard as true certain sentences which, according to this semantic scheme, should lack truth value. Tappenden tried to solve this problem by using a new speech act, articulation. Unlike assertion, which implies truth, articulation only implies non-falsity. In this paper I argue that Tappenden's strategy cannot be successfully applied to truth and the Liar.
\end{abstract}

Keywords

Paradoxes, truth, vagueness, Sorites, the Liar.

\section{Introduction}

In The Liar and Sorites Paradoxes: Toward a Unified Treatment (Tappenden 1993), Jamie Tappenden suggests a line of thought according to which vague predicates and the truth predicate are similar enough to support a special speech act which he calls 'articulation'. The common treatment of these two phenomena, claims Tappenden, would constitute a first step towards a unified solution to the Liar and the Sorites paradoxes.

In principle, the Liar and the Sorites paradoxes seem totally unrelated. First, self-reference (in some or other fashion) seems to play a crucial role in the former but not in the latter; second, on the other hand, truth does not seem to be a vague predicate. Nevertheless, some authors have tried to offer a common solution to the two paradoxes. In fact, Tappenden was one of the first to do so. 
Among the reasons why we should expect a common solution to these two paradoxes, we might consider a group of explanations related to methodological issues such as simplicity and uniformity. It might be argued that, in general, it is worth seeking common solutions to different paradoxes because this would be a way to deal with all of them while using a minimum of resources. Hence, in particular, it is worth seeking a common solution to the Liar and the Sorites. ${ }^{1}$

In the second place, sometimes a certain group of paradoxes are taken to be of the same kind and, hence, they should be treated, it is claimed, in the same way. Following this line of thought, Graham Priest has defended that paradoxes that somehow involve self-reference (the Liar among them) all have a common underlying structure that is captured by what he calls 'the inclosure schema' (see, specially, Priest 2002 and Priest 1994). More recently, Priest (2010) has proposed that the Sorites also satisfies this schema, which means that both the Liar and the Sorites have a common underlying structure and, hence, according to him, they should be treated uniformly. ${ }^{2}$

Instead of following Priest's direct strategy and describing the underlying structure of both the Liar and the Sorites, some authors have followed a more indirect path and have offered evidence in favour of the idea that the two paradoxes share some structural properties. We find this idea applied specifically to the Liar and the Sorites in the work of Jamie Tappenden, who, as we are going to see, draws attention to the fact that one can decide somewhat arbitrarily the extension of both vague predicates and the truth predicate. ${ }^{3}$

To my mind there is enough evidence to seriously entertain the possibility of a common treatment to the Liar and the Sorites. In this paper I will examine and discuss whether Tappenden's ideas stemming from vagueness can be successfully applied to the Liar case; and I will eventually defend that they cannot.

${ }^{1}$ This idea can be found, for example, in Hyde 2013.

${ }^{2}$ See, especially, Priest 1994: 32 and Priest 2002: 166.

${ }^{3}$ Another important structural similarity between vagueness and truth that has been stressed in the literature is the fact that in both cases some kind or other of indeterminacy is involved. This idea is defended by Tappenden himself and can also be found in many places like, for example, McGee 1991, Field 2003, Field 2008, Priest 2010 or Hyde 2013. 
The general framework Tappenden accepts for truth and the Liar is Kripke's proposal (see Kripke 1975) built with the Strong Kleene semantic scheme for the logical constants. The Strong Kleene scheme preserves what Tappenden calls 'the truth-functional intuition', according to which the truth value of a complex sentence built with a logical connective should depend on the truth value of its parts. Furthermore, Tappenden applies this scheme to vagueness and the Sorites and, using the supervaluationist framework, he gives an explanation of the existence of what he calls 'the penumbral intuition'; that is, the intuition underlying the idea that there are certain sentences which we are strongly inclined to consider as true but which, according to the truth-functional intuition, lack truth value.

The structure of the paper is as follows. In the next two sections, I will introduce Tappenden's view on vagueness and the Sorites. Next, in the fourth and fifth sections I will present and discuss Kripke's account of the Liar and its application to Tappenden's framework.

\section{Intuitions and sharpenings}

Tapppenden (1993) presents two contrasting intuitions that underlie the phenomenon of vagueness: the truth-functional and the penumbral intuitions. According to the former, we tend to see logical connectives as truth functions, in the sense that the truth value of a sentence with a given logical connective depends on the truth value of its parts. Moreover, this value should be uniform; that is, if sentences $\varphi$ and $\psi$ have the same form and their sentential constituents have the same truth values, then $\varphi$ and $\psi$ should share the same truth value. Consequently, according to the truth-functional intuition, the truth value of a sentence like 'if John is tall, then Joe is' will depend on the truth value of the sentences 'John is tall' and 'Joe is tall'. Thus, for example, the Strong Kleene scheme (SK henceforth), which preserves the truth-functional intuition, will declare the sentence 'if John is tall, then Joe is' to be gappy (neither true nor false) ${ }^{4}$ when both John and Joe are borderline cases of being tall, so that both

\footnotetext{
${ }^{4}$ Since Tappenden uses the term 'indeterminate', as we shall see, in a non standard way, I will follow Oms (2010) and I will refer to sentences that are neither true nor false as 'gappy' instead of 'indeterminate'.
} 
'John is tall' and 'Joe is tall' are gappy.

In contrast, according to the penumbral intuition, there are sentences that seem almost analytic to us and such that we are strongly inclined to assign truth to them, even if, according to the truth-functional intuition, they might be gappy. To see this, consider again the sentence 'if John is tall, then Joe is' in a situation where, again, both John and Joe are borderline cases of being tall (and, consequently, both 'John is tall' and 'Joe is tall' are gappy) but where Joe is taller than John. In this case, we strongly tend to assign truth to 'if John is tall, then Joe is'. Tapenden (1993), following Fine (1975), calls sentences like this last one 'penumbral sentences'.

Specifically, a penumbral sentence is, according to Tappenden, a particular case of a broader type of sentences which he calls 'preanalytic sentences'. In order to characterize pre-analytic sentences, Tappenden uses the supervaluationist notion of complete admissible precisification. Vague predicates are such that their extensions can be made precise in several ways. This does not mean, though, that all increases in precision are equally admissible; sometimes, the meaning of the predicate whose extension is being made precise puts constraints on which increases in precision are acceptable. To see this, consider, following Tappenden, the predicate 'tung' whose meaning is determined by the following rules:

(a) 'tung' applies to anything of mass greater than $200 \mathrm{Kg}$.

(b) 'tung' does not apply to anything of mass less than $100 \mathrm{Kg}$.

Apparently, we could understand 'heavy' in a way similar to 'tung'; we could say that anything of mass greater than $200 \mathrm{Kg}$ would count as being heavy and that anything of mass less than $100 \mathrm{Kg}$ would count as not being heavy. Even in this case, though, there would be a crucial difference between 'tung' and 'heavy'. In the case of the former, given (a) and (b), nothing prevents us from further increasing the precision of 'tung' in such a way that, given two objects $A$ and $B$, $B$ heavier than $A$ and both unsettled with respect to the predicate, $B$ counts as non-tung while $A$ counts as tung. But we cannot precisify 'heavy' (that is, make it more precise) in this way; if $B$ is heavier than $A$, then the meaning of 'heavy' implies that, if $A$ is heavy, so is $B$. We 
can say, then, that a precisification (a way of precisifying a predicate) ${ }^{5}$ is admissible if the more sharply drawn boundaries are acceptable according to the meaning of the predicate. Furthermore, a precisification is complete if it is classical, that is, if it decides all the possible cases. Supervaluationism uses complete admissible precisifications in order to establish some of the semantic properties of the sentences with vague expressions. ${ }^{6}$

These constraints on increases in precision can be understood of as assignments of truth values to certain sentences. In Tappenden's own words:

The above discussion suggests one collection of constraints on increases of precision: those which may be formulated, "Never make words $w_{1}, \ldots . ., w_{\mathrm{n}}$ more precise in such a way that sentence $S$ become false". (Tappenden 1993: 557)

Sentences like $S$ are called by Tappenden 'pre-analytic':

[A] sentence $S$ is pre-analytic if anyone who understands $S$ knows not to draw more precise boundaries to any of the expressions in $S$ in such a way that $S$ would be false in any circumstances. Intuitively, pre-analytic sentences are like analytic sentences, except that analytic sentences are always true, while pre-analytic sentences are never false. (Tappenden 1993: 557)

Sentences like the ones above, 'if John is tall, then Joe is' (in the intended situation), would be examples of pre-analytic sentences in Tappenden's sense. As he notes in the above quote, pre-analytic sentences are never false but, depending on the semantic frame, they do not need to be always true. Once we have the precisification machinery we can also characterize a new kind of sentence, the indeterminate sentences, which are true in some complete admissible precisification and false in some other. ${ }^{7}$

Penumbral sentences are, according to Tappenden, pre-analytic

${ }^{5}$ Precisifications are called 'elaborations' by Tappenden. I will follow the standard nomenclature and I will use 'precisification' instead of 'elaboration'.

${ }^{6}$ The classical paper on the supervaluationist view is Fine 1975. See McGee 1991 or Keefe 2000 for some applications of the supervaluationist ideas.

${ }^{7}$ Note that, clearly, being indeterminate is not the same as being neither true nor false (gappy), for nothing prevents pre-analytic sentences from being gappy too. 
sentences that, relative to some semantic assignment respecting the truth-functional intuition, are considered neither true nor false, even though we are strongly inclined to regard them as true. We will define them in more detail in the following section.

\section{Vagueness}

As I said, Tappenden (1993) defends an approach to vagueness and the Sorites that uses SK. More specifically, Tappenden uses a partial model, called the 'pre-assignment', that assigns to any predicate $P$ its extension, that is, the set of objects to which the predicate clearly applies, and its anti-extension, that is, the set of objects to which the predicate clearly fails to apply. Vagueness is captured by the fact that the extension and the anti-extension of the vague predicates may not be exhaustive, so that some cases may be neither in the extension nor in the anti-extension. Tappenden then defines having semantic value 1 (for our present purposes we can consider that having semantic value 1 is just being true) in the pre-assignment using SK. A sentence, then, is true if it is true in the pre-assignment and false if it is false in the pre-assignment. The truth-functional intuition, hence, plays a central role in Tappenden's picture.

One immediate problem for this account is that it cannot distinguish between predicates like 'tung' and 'heavy'; that is, it cannot express how constraints on increases in precision are embodied in the meaning of predicates. This can easily be seen if we consider two borderline objects $A$ and $B$ of both 'tung' and 'heavy'. If we suppose that the extension and the anti-extension that the pre-assignment assigns to 'heavy' are the same as the ones assigned to 'tung', it is clear that 'if $A$ is tung so is $B$ ' has the same status as the sentence 'if $A$ is heavy so is $B$ '; both are gappy. This makes it hard to see how we could express the intuitive difference in meaning between the predicates 'tung' and 'heavy'. The problem is that we cannot show that 'tung' and 'heavy' can be precisified in different ways and, since we are supposing that both have the same extension and the same antiextension, we cannot tell them apart.

At this point, the supervaluationist machinery comes to the rescue. Recall that indeterminate sentences are gappy sentences that 
are true in some complete admissible precisification ${ }^{8}$ and false in some other. Penumbral sentences, in contrast, are sentences that are neither true nor false in the pre-assignment and such that they are never false in any complete admissible precisification, to wit they are gappy, but also they are never false. Consequently, penumbral sentences are not indeterminate. Then, in a situation where $A$ and $B$ are two borderline objects of both 'tung' and 'heavy' and, moreover, $B$ is heavier than $A$, both sentences 'if $A$ is tung so is $B$ ' and 'if $A$ is heavy so is $B$ ' are gappy; but the first is indeterminate while the second is penumbral, so we are able to distinguish the differences in the semantic behavior between the two predicates.

To sum up, according to Tappenden, sentences can have the following semantic statuses; they can be true (that is, true in the pre-assignment), false (false in the pre-assignment) or gappy (they can lack truth value). Among gappy sentences we can distinguish between indeterminate sentences (if there is a precisification where they are true and a precisification where they are false) and penumbral sentences (they form a subset of the pre-analytic sentences and are never false in any precisification).

The supervaluationist apparatus also allows Tappenden to account for the penumbral intuition. And it is here that a new speech act, articulation, plays its role. Recall that the problem is that the sentence 'if John is tall, then Joe is' (in the intended situation) is gappy and, hence, it is not true. Still, we are strongly inclined to regard it as true; that is what the penumbral intuition is about.

As I said, Tappenden's answer appeals to a new speech act. Tappenden asks us to consider the fact that the use of natural languages is a very complex phenomenon and, consequently, it can easily degenerate into a confusion of tongues. That is why we need to maintain the stability of the conventions of a language by correcting the linguistic mistakes of other speakers. Tappenden's insight is that such corrections are typically made with the use of pre-analytic sentences. Thus, if you hear somebody who, knowing Joe is taller than John, utters 'John is tall and Joe is not', then you, in order to show her that she has not correctly grasped the meaning of the word 'tall' and to

${ }^{8}$ From now on, when the context allows it, I will simply use 'precisification' to refer to complete admissible precisifications. 
correct her mistake, will utter 'if John is tall, then Joe is'. According to Tappenden, the general pattern of this activity is the following one:

[W] utter a declarative sentence $S$ in order to induce the withdrawal of a mistaken utterance of $\neg S$, or the withdrawal of other utterances that can only be true if $\neg S$ is true, or to ward off anticipated mistaken utterances of $\neg S$, by indicating that $\neg S$ is never correctly assertable. (Tappenden 1993: 570)

The idea behind articulation is the following. Consider these two claims:

(i) A condition of correctness for a literal assertion of a sentence $\varphi$ is that $\varphi$ must be true.

(ii) If $\neg \varphi$ is true, then $\varphi$ is false and, consequently, if $\varphi$ is not false, then $\neg \varphi$ is not true.

From (i) and (ii) it follows that it is sufficient to show that $\varphi$ is not false in order to show the incorrectness of the assertion of $\neg \varphi$. This means that a sentence used to correct a linguistic mistake must be non-false in order to imply that its negation is not true and, consequently, not assertable. When a sentence $\varphi$ is used in this way to correct a mistaken utterance of $\neg \varphi$, Tappenden says that $\varphi$ has been articulated, not asserted. The main difference is that, while assertion implies truth, articulation only implies non-falsity. Then, according to Tappenden, penumbral sentences are typically articulated and, therefore, do not need to be true, but only non-false - which they are, as they are not false in any precisification. If we sometimes mistakenly judge that they can assert something and, hence, that they need to be true, this is because we are confused about assertion and articulation. This confusion is due to the fact that the behavior by which its goals are attained (that is, in the case of assertion, to say something about the world, and, in the case of articulation, to correct a linguistic mistake) is the same; but it is the same by a happy coincidence.

In this paper I will not look any further into Tappenden's approach to vagueness. What I intend to do, in the next sections, is to see whether he can successfully apply the strategy we have just seen 
for vagueness to the case of truth and the Liar paradox. ${ }^{9}$

\section{Truth and fixed points}

As I said, Tappenden endorses Kripke's approach (with SK) to the problem posed by the Liar paradox. In his seminal paper Outline of a Theory of Truth (Kripke 1975), Saul Kripke presented one of the most influential approaches to the Liar paradox. The goal Kripke wants to achieve is a language with its own truth predicate; that is, he wants a language with a certain monadic predicate, $T r$, that can be applied to sentences containing $T r$ itself and such that can be said to appropriately capture the notion of truth. As a sufficient condition to capture the notion of truth, we can take the satisfaction of the Intersubstitutivity Principle (IP):

(IP) If two sentences $\varphi$ and $\psi$ are alike except that one has a sentence $\chi$ where the other has $\operatorname{Tr}\ulcorner\chi\urcorner$, then $\varphi$ and $\psi$ have the same semantic value. ${ }^{10}$

The problem is that the sentence $\lambda$ (let us call it 'the Liar sentence') identical to the negation of its own truth ascription (so that $\lambda \leftrightarrow \neg \operatorname{Tr}\ulcorner\lambda\urcorner$ holds) shows that this goal cannot be achieved in classical logic. This is the main lesson of the Liar paradox. To see this, note that, by IP and the construction of $\lambda$, we can easily obtain $\operatorname{Tr}\ulcorner\lambda\urcorner \leftrightarrow \neg \operatorname{Tr}\ulcorner\lambda\urcorner$, which, in classical logic, is equivalent to a plain contradiction: $\operatorname{Tr}\ulcorner\lambda\urcorner \wedge \neg \operatorname{Tr}\ulcorner\lambda\urcorner$.

In a well-known passage, Kripke describes the intuition underlying his proposal:

We wish to capture an intuition of somewhat the following kind. Suppose we are explaining the word 'true' to someone who does not yet understand it. We may say that we are entitled to assert (or deny) of any sentence that it is true precisely under the circumstances when we can

\footnotetext{
${ }^{9}$ For some criticisms of Tappenden's view on vagueness and the Sorites, see Graff Fara 2000, Keefe 2000 and Oms 2010.

${ }^{10}$ Here, the brackets (' $\ulcorner$ ' and ' $\urcorner$ ') indicate some device of canonical name formation for sentences; thus, ' $\ulcorner\varphi\urcorner$ ' is just a name for the sentence $\varphi$ and ' $\operatorname{Tr}\ulcorner\varphi\urcorner$ ' ascribes truth to the sentence $\varphi$. Kripke did not use this principle, I am following Hartry Field's formulation of Kripke's theory (see, for instance, Field 2008). IP is claimed to hold, of course, only in non-opaque contexts.
} 
assert (or deny) the sentence itself. Our interlocutor then can understand what it means, say, to attribute truth to (6) ('snow is white') but he will still be puzzled about attributions of truth to sentences containing the word 'true' itself. [...] (Kripke 1975: 701)

The idea is that, once the subject who is learning the meaning of 'true' can assert 'snow is white' is true', she will be entitled to assert "'snow is white' is true' is true', and so on. This process can be repeated so that

the subject will eventually be able to attribute truth to more and more statements involving the notion of truth itself. There is no reason to suppose that all statements involving 'true' will become decided in this way, but most will. Indeed, our suggestion is that "grounded" sentences can be characterized as those which eventually get a truth value in this process. (Kripke 1975: 701)

The idea behind Kripke's proposal, hence, is that the semantic status of an ascription of truth to a sentence $\varphi, \operatorname{Tr}\ulcorner\varphi\urcorner$, will be established once the semantic status of $\varphi$ itself is established. The process described in the quote will eventually evaluate many sentences containing 'true', but some others, like the Liar, will remain undecided.

In order to make this idea precise Kripke proposes considering an interpreted first-order language $\mathcal{L}$, the base language, as an idealization of the natural language without the truth predicate. Next, Kripke shows how that base language can be expanded with a truth predicate, $T r$, in a way that we achieve a fixed-point, that is, an extension of $\operatorname{Tr}$ that contains the very true sentences we obtain by having it as extension of $T r$. In more detail, let us have a classical model $\mathcal{N}$ for the base language (without $T r$ ), and use $|\varphi|_{\mathcal{N}, E}$ to refer to the semantic value, using SK, of the sentence $\varphi$ in $\mathcal{N}$ when the extension of Tr is $E$. Then, by the use of a transfinite construction, Kripke shows how to construct a set of sentences, $K$, which is a fixed point of the construction; that is, for any sentence $\varphi$ of the expanded language with $\operatorname{Tr}$, the semantic value of $\varphi$ is always identical to the semantic value of its truth ascription: $|\operatorname{Tr}\ulcorner\varphi\urcorner|_{\mathcal{N}, K}=|\varphi|_{\mathcal{N}, K}$. Kripke calls the sentences in $K$ and the ones whose negation is in $K$ 'grounded', and the remaining sentences 'ungrounded'. This means that the Liar is ungrounded.

$K$ is not the only fixed point that can be constructed over a given base model. As a matter of fact, $K$ is the minimal fixed point; there are 
many different fixed points that can be achieved and $K$ is the smallest one, in the sense that it is included in all the others. One way to see this is to consider, for example, the Truth-teller, a sentence $\tau$ that is identical to its own truth ascription:

( $\tau) \quad \operatorname{Tr}\ulcorner\tau\urcorner$

Notice that we can freely ascribe any truth value we want to $\tau$; that is, if $\tau$ is true, then, since that is precisely what $\tau$ says, it is indeed true. But if it is false, then the negation of $\tau$ holds and, consequently, it is, indeed, false. This means that we can have at least three fixed points: $K$, in which $\tau$ is ungrounded, and two more, one that assigns truth to $\tau$ and another one that assigns truth to $\neg \tau .^{11}$ Although Kripke does not commit himself to any particular fixed point, the minimal fixed point $K$ is usually considered the most natural candidate as the extension of the truth predicate. ${ }^{12}$

One of the main problems of Kripke's approach with SK is that the resulting theory is too weak, as SK fails to validate some elementary laws such as $\varphi \rightarrow \varphi$. Actually, it is well-known that SK has no tautologies at all.

One of the main consequences of this fact is that, although Kripke's construction with SK satisfies IP, it cannot satisfy the T-schema: ${ }^{13}$

(T-schema) $\quad \varphi \leftrightarrow \operatorname{Tr}\ulcorner\varphi\urcorner$

By the SK interpretation of the conditional, it is clear that when $\varphi$ is the Liar sentence, none of the directions of the biconditional in the T-schema is true.

\section{The Liar}

Let us begin this section by presenting the problem we just saw about the weakness of SK in a different way. Recall that the Liar reasoning allows us to conclude that the Liar is true if, and only if, it is not

\footnotetext{
${ }^{11}$ For details on the fixed points above the minimal one, see, for example, Visser 1989 or Gupta and Belnap 1993.

${ }^{12}$ See, for instance, Soames 1999 or Kremer 1988.

${ }^{13}$ Tarski defended that any materially adequate theory of truth should imply all the instances of the T-schema (see, for instance, Tarski 1983).
} 
true. Now imagine yourself explaining this paradox to someone who does not seem to understand it. As Tappenden observes, in a situation like that,

[y]ou might well say: "Here is what is funny about [the Liar]. If [it] is true, then it is not true, and if [it] is not true then it is true". (Tappenden 1993: 552)

And I would add: 'But that is impossible, for no sentence can be both true and not true'. Although the sentence in the quote, $\operatorname{Tr}\ulcorner\lambda\urcorner \leftrightarrow \neg \operatorname{Tr}\ulcorner\lambda\urcorner$ (its formal counterpart), might seem contradictory, it is the appropriate thing to say in this situation; we seem to be asserting it appropriately. And the same happens with the second sentence, $\neg(\operatorname{Tr}\ulcorner\lambda\urcorner \wedge \neg \operatorname{Tr}\ulcorner\lambda\urcorner)$, which is gappy in Kripke's construction, which implies that it should not be assertable. This is just a consequence of the weakness of SK. This situation is taken by Tappenden to be a point in favor of applying the strategy he has used in the case of vagueness and the Sorites to the case of truth and the Liar (Tappenden 1993:574-77).

Specifically, Tappenden's strategy consists in defending that 'some of the considerations that pertained to vague predicates may be carried over' (Tappenden 1993: 575) to the truth predicate, so that articulation can be used in order to explain why certain sentences that have no truth value according to Kripke's approach seem true to us; that is, Tappenden wants to explain the penumbral intuition applied to the truth predicate. Some of the sentences Tappenden thinks fall under the penumbral intuition are, among others, the sentence $\neg(\operatorname{Tr}\ulcorner\lambda\urcorner \wedge \neg \operatorname{Tr}\ulcorner\lambda\urcorner)$ of the previous example, instances of the Law of Excluded Middle applied to ungrounded sentences and, of course, the instances of the T-schema of ungrounded sentences. All these sentences are pre-analytic; that is, they are never false, in a sense to be specified shortly. Besides, since they are gappy in SK, they are penumbral sentences.

Let us, first, look at the features of the truth predicate that make Tappenden claim that we can carry over to truth the results we obtained about vagueness. The main such feature Tappenden puts forward is the arbitrariness that can be found when we try to determine the extension of the truth predicate. Specifically, Tappenden mentions the Truth-teller, the sentence $\tau$ (introduced in the previous 
section) which is identical to its own truth ascription. As we saw, and so Tappenden claims, we can consistently assign $\tau$ to the extension of $\operatorname{Tr}$ and we can also consistently assign $\neg \tau$ to this extension. This suggests, according to him, that there will exist constraints on how these different stipulations of the extension of the truth predicate might be. Among these constraints there will be sentences like 'for any sentence $x, \operatorname{Tr}\ulcorner x\urcorner \vee \neg \operatorname{Tr}\ulcorner x\urcorner$ ' or, indeed, any sentence of the form $\operatorname{Tr}\ulcorner\varphi\urcorner \leftrightarrow \varphi$. These sentences are evaluated as gappy in Kripke's framework and, hence, they are not assertable. Why, then, in situations like the one described above, do we seem to assert them? Why do they seem true to us? How can we explain the penumbral intuition applied to truth? Here is where Tappenden uses the notion of articulation:

As with vague predicates, we may explain away the penumbral intuition by noting the way the patterns of use of the relevant sentences lead us to take them unreflectively to be true. The Tarski biconditional with liar instances [...] might well be uttered in the course of an attempt to demonstrate why it is unacceptable to assert [the Liar] and unacceptable to assert the negation of [the Liar]. The imagined utterance of Tarski biconditional is successful if the hearer recognizes that certain other sentences cannot be correctly asserted; so the account of articulation [...] extends naturally to this expanded setting. (Tappenden 1993: 576)

Recall now how articulation worked: essentially, we uttered a sentence $\varphi$ in order to show that it was unacceptable to assert $\neg \varphi$. But since in order to show that $\neg \varphi$ cannot be asserted it is enough to show that it is not true, and since $\varphi$ being not false implies that $\neg \varphi$ is not true, we concluded that showing that $\varphi$ is not false is enough to show that $\neg \varphi$ cannot be asserted. Now, in the case of vagueness, pre-analytic sentences were typically articulated, according to Tappenden, because they were never false in any precisification. Can we adapt this idea to truth? I do not think so.

As I said in the presentation of Kripke's approach, $K$ is just the minimal fixed point; there are many other fixed points that extend $K$ and that, as fixed points, validate IP (so that they can be claimed to be possible extensions for Tr). Kripke (1975) also showed that every fixed point can be extended to a maximal fixed point, where a maximal fixed point, in Kripke's words, is 'a fixed point that has 
no proper extension that is also a fixed point. Maximal fixed points assign "as many truth values as possible"; one could not assign more consistently with the intuitive concept of truth' (Kripke 1975: 708).

Now, if Tappenden's use of articulation applied to the Liar is to make sense, the instances of the T-schema will be pre-analytic sentences; that is, sentences that cannot be evaluated as false in any way in which the truth predicate is made precise. What this means is that the most natural candidates for being the admissible ways to make the truth predicate precise are the fixed points, because they never make any instance of the T-schema false. Recall, though, that, apart from being admissible, the precisifications of the vague predicates had to be complete; all cases had to be decided. In the case of truth, this is not possible, if the instances of the T-schema are regarded as pre-analytic. To see why, suppose $X$ is a complete way of making the truth predicate precise and $\mathcal{N}$ is a model for the base language $\mathcal{L}$ as introduced in the previous section. Now, if $X$ is complete, either $|\lambda|_{\mathcal{N}, X}=1$ or $|\lambda|_{\mathcal{N}, X}=0$. Suppose $|\lambda|_{\mathcal{N}, X}=1$ (the other case is analogous). Then, by definition of $\lambda,|\neg \operatorname{Tr}\ulcorner\lambda\urcorner|_{\mathcal{N}, X}=1$ and, hence, $|\operatorname{Tr}\ulcorner\lambda\urcorner|_{\mathcal{N}, X}=0$. Consequently, $|\operatorname{Tr}\ulcorner\lambda\urcorner \leftrightarrow \lambda|_{\mathcal{N}, X}=0$. So, if the truth predicate is made completely precise some instances of the T-schema will be false and, hence, the T-schema will not count as pre-analytic. That means that the ways of making the truth predicate precise cannot be complete, which, in turn, means that classical logic cannot be used in them, in sharp contrast to vagueness. It is to be expected, then, that in order to evaluate the semantic value of the sentences in the fixed points, we will have to use SK.

Hence, the picture we are unraveling is the following one. The semantic value of the sentences involving truth is determined by $K$, the minimal fixed point. Then, some sentences that have no truth value are such that we are strongly inclined to believe them true. This inclination, though, is an illusion prompted by the fact that, although they do not have truth value, they cannot be false, which is what is needed to correct linguistic mistakes. Now, since given that they are never false and, hence, they are used to correct linguistic mistakes and given that correcting linguistic mistakes with such sentences (articulating them) is so similar to asserting them, we mistakenly conclude that they are asserted, which in turn implies that they are true. In order to make sense of the idea of a sentence with vague 
predicates that cannot be false, Tappenden used the supervaluationist machinery. Then, the suggestion is to make a similar move for the truth predicate; pre-analytic sentences involving truth will be sentences that are not false in any way of making the truth predicate precise that does not conflict with its meaning, that is, sentences that are not false in any fixed point. The fixed points, though, are not complete precisifications of the truth predicate; they cannot be if we want the instances of the T-schema to be pre-analytic.

But the fact that the ways of making the truth predicate more precise are not complete is fatal for, although it is true that sentences like (i) 'for any sentence $x, \operatorname{Tr}\left\ulcorner_{x}\right\urcorner \vee \neg \operatorname{Tr}_{r}\left\ulcorner_{x}\right.$ ' are never false in any fixed point, the same happens, for example, to (ii) 'for some sentence $x$, $\operatorname{Tr}\left\ulcorner{ }^{\prime}\right\urcorner \wedge \neg \operatorname{Tr}\left\ulcorner{ }^{\prime}\right\urcorner$ '. To see this, notice that, since the fixed points are not complete, there will always be at least one ungrounded sentence, say, $\varphi$. Then, by the SK's rules, $\operatorname{Tr}\ulcorner\varphi\urcorner \wedge \neg \operatorname{Tr}\ulcorner\varphi\urcorner$ will be gappy, which, in turn, implies the gappiness of (ii). Consequently, (ii) will be gappy in all the fixed points and, hence, it will never be false.

This means that if we characterize pre-analytic sentences as sentences that are never false in any way of making 'true' precise, (ii) will be pre-analytic. But pre-analytic sentences were supposed to capture some important features of the meaning of the predicates involving them, which in the case of (ii) is clearly not the case. Moreover, (ii) could be, in consequence, successfully articulated, which is absurd. This means that Tappenden must provide us with a new characterization of pre-analytic sentences; but it is not at all clear how it might proceed..$^{14,15}$

\footnotetext{
${ }^{14}$ The truth predicate becomes, then, something on the lines of what Tappenden calls 'an essentially vague predicate'. The idea is that there are predicates (like 'roughly heavy', 'roughly within walking distance of Barcelona' or 'roughly a handful of sand') that are essentially vague, in the sense that they do not accept complete precisifications in virtue of their meaning. Other authors like, for example, Eklund (2001) propose similar predicates. For both Tappenden and Eklund, such predicates present a serious problem to Supervaluationism because they challenge the rationale for singling out a particular set of precisifications as acceptable. As Oms (2010) notes, though, essentially vague predicates also jeopardize Tappenden's position, for, given the way he uses the supervaluationist machinery, such predicates seem to make it impossible for him to distinguish between the predicates 'roughly tung' and 'roughly heavy'.

${ }^{15}$ I would like to thank José Martínez Fernández, Elia Zardini and an anonymous
} 


\section{References}

Eklund, Matti. 2001. Supervaluationism, vagueifiers, and semantic overdetermination. Dialectica 55: 363-378.

Field, Hartry. 2003. The semantic paradoxes and the paradoxes of vagueness. In Liars and Heaps, New Essays on Paradox. Edited by J.C. Beall. Oxford: Oxford University Press.

Field, Hartry. 2008. Saving Truth from Paradox. Oxford: Oxford University Press. Fine, Kit. 1975. Vagueness, truth and logic. Synthese 30: 265-300.

Graff Fara, Delia. 2000. Shifting sands. Philosophical Topics 28: 45-81.

Gupta, Anil and Nuel Belnap. 1993. The Revision Theory of Truth. Cambridge, Mass.: MIT Press.

Hyde, Dominic. 2013. Are the sorites and liar paradox of a kind? In Paraconsistency: Logic and Applications. Edited by Francesco Berto et al. Logic, Epistemology and the Unity of Science 26: 349-366.

Keefe, Rosanna. 2000. Theories of Vagueness. Cambridge: Cambridge University Press.

Kremer, Michael. 1988. Kripke and the logic of truth. Journal of Philosophical Logic 17: 225-278.

Kripke, Saul A. 1975. Outline of a theory of truth. Journal of Philosophy 72: 690-716.

McGee, Vann 1991. Truth, Vagueness, and Paradox. Indianapolis: Hackett Publishing Company.

Oms, Sergi. 2010. Truth-functional and penumbral intuitions. Theoria 25: 137-147.

Priest, Graham. 1994. The structure of the paradoxes of self-reference. Mind 103: $25-34$

Priest, Graham. 2002. Beyond the Limits of Thought. Oxford: Oxford University Press.

Priest, Graham. 2010. Inclosures, vagueness, and self-reference. Notre Dame Journal of Formal Logic 51: 69-84.

Soames, Scott. 1999. Understanding Truth. Oxford: Oxford University Press. Tappenden, Jamie. 1993. The liar and sorites paradoxes: toward a unified

referee for all their very stimulating comments. During the writing of the paper, I have benefitted from the project FFI2015-70707P of the Spanish Ministry of Economy, Industry and Competitiveness on Localism and Globalism in Logic and Semantics. 
treatment. Journal of Philosophy 60: 551-577.

Tarski, Alfred. 1983. The concept of truth in formalized languages. In Logic, Semantics, Metamathematics. Edited by John Corcoran. Translated by J. H. Woodger. Indianapolis: Hackett Publishing Company.

Visser, Albert. 1989. Semantics and the liar paradox. In Handbook of Philosophical Logic 4:617-706. 